\title{
RIGOROUS SOLUTION OF A MEAN FIELD SPIN GLASS MODEL
}

\author{
T.C. DORLAS \\ University of Wales Swansea \\ Department of Mathematics \\ Swansea SA2 8PP UK \\ E-mail: T.C.Dorlas@wsansea.ac.uk \\ J.R. WEDAGEDERA ${ }^{1}$ \\ University of Ruhuna \\ Department of Mathematics \\ Matara, Sri Lanka \\ E-mail: janak@maths.ruh.ac.lk
}

(Received March, 1998; Revised December, 1999)

A separable spin glass model whose exchange integral takes the form $J_{i j}=J\left(\xi_{i 1} \xi_{j 2}+\xi_{12} \xi_{j 1}\right)$ which was solved by van Hemmen et al. [12] using large deviation theory [14] is rigorously treated. The almost sure convergence criteria associated with the cumulant generating function $C(t)$ with respect to the quenched random variables $\xi$ is carefully investigated, and it is proved that the related excluded null set $\mathcal{N}$ is independent of $t$. The free energy and hence the other thermodynamic quantities are rederived using Varadhan's Large Deviation Theorem. A simulation is also presented for the entropy when $\xi$ assumes a Gaussian distribution.

Key words: Spin Glass, Large Deviations, Egodicity.

AMS subject classifications: $82 \mathrm{C} 31,82 \mathrm{~B} 31,82 \mathrm{D} 99$.

\section{Introduction}

In this paper, an exactly solvable model of a spin glass which was introduced by van Hemmen et al. $[12,13]$ is studied. The Hamiltonian function is given by

$$
\mathfrak{H}_{N}(\{S\},\{J\})=-\sum_{(i, j)} J_{i j} \omega(i) \omega(j)-J_{0} \sum_{(i, j)} \omega(i) \omega(j)-h \sum_{i} \omega(i),
$$

where $\omega(i)= \pm 1$ are $N$ Ising spins interacting with each other in pairs $(i, j)$ and with an external magnetic field $h . J_{0}$ is a direct ferromagnetic coupling and

\footnotetext{
${ }^{1}$ Supported by the Commonwealth Scholarship Commission, United Kingdom.
} 


$$
J_{i j}=\frac{J}{N}\left[\xi_{i} \eta_{j}+\xi_{j} \eta_{i}\right]
$$

where the $\xi_{i}$ and $\eta_{j}$ are i.i.d. random variables with a symmetric distribution with zero mean and variance 1 . This model resembles a similar model proposed by Pastur and Figotin in [8-10]. However, it was van Hemmen et al. who first used a large deviation (LD) argument to successfully solve this model. In the absence of ferromagnetic coupling and an external magnetic field $h$, the Hamiltonian can also be written as

$$
\mathfrak{H}_{N}(\{S\},\{J\})=-N J\left[N^{-1} \sum_{i=1}^{N} \xi_{i} \omega(i)\right]\left[N^{-1} \sum_{j=1}^{N} \eta_{j} \omega(j)\right]
$$

To compute, using LD theory, the free energy defined at inverse temperature $\beta$ by

$$
-\beta f(\beta)=\lim _{N \rightarrow \infty} \frac{1}{N} \log \sum_{\{\omega(i)\}_{i=1}^{N}} e^{-\beta^{3} \mathcal{G} N}
$$

it must be shown that the so-called cumulant generating function exists, defined by

$$
C(t)=\lim _{N \rightarrow \infty} \frac{1}{N} \log \mathbb{E}\left[\exp \left\langle t, W_{N}\right\rangle\right]
$$

where $\mathbb{E}$ denotes a configuration average over the Ising spins $\omega(i),\langle-,-\rangle$, represents an Euclidean inner product in $\mathbb{R}^{2}$ and $W_{N}$ are the 2 -dimensional random variables

$$
W_{N}=\left(\frac{1}{N} \sum_{i=1}^{N} \xi_{i} \omega(i), \frac{1}{N} \sum_{i=1}^{N} \eta_{i} \omega(i)\right) \text {. }
$$

The focus of this paper is to rigorously investigate the a.s. convergence criteria of $C(t)$ with respect to the common distribution of $\xi$ and $\eta$. The main result is Theorem 3.1 in which the $t$ independency of the excluded null set associated with the above mentioned convergence result is proved. Furthermore, we show in Proposition 3.2 that

(i) when $\xi$ has a general symmetric discrete distribution, then the entropy density $s$ satisfies

and

$$
s \rightarrow \mathbb{P}\left(\xi_{1}+\xi_{2}=0\right) \ln 2 \quad \text { as } T \rightarrow 0
$$

(ii) when $\xi$ has a Gaussian distribution, $s \rightarrow 0$ as $T \rightarrow 0$.

\section{Definitions}

Consider the configuration space $\Omega_{\Lambda}=X^{\Lambda}$ (i.e., $\Omega_{\Lambda}=\{\omega: \Lambda \rightarrow X\}$ ), where $X$ is a compact metric space, and $\Lambda=\{1, \ldots, N\}$ where $N$ is any non-negative integer. In this particular case, we take $X=\{-1,1\}$. Define the single spin distribution $\mu$ by

$$
\mu=\frac{1}{2}\left(\delta_{1}+\delta_{-1}\right)
$$


so that $\mu(\{1\})=\mu(\{-1\})=\frac{1}{2}$. Here $\delta_{(-)}$is the Dirac-point measure. Also denote the infinite product measure on $\mathfrak{B}\left(\Omega_{\Lambda}\right)$ (the Borel subsets of $\Omega_{\Lambda}$ ) with identical onedimensional marginals $\mu$ by $\mathbb{P}_{\mu}(\omega)=2^{-|\Lambda|}$ for each $\omega \in \Omega_{\Lambda}$. Define a random interaction $\Phi=\left\{\Phi_{\Lambda}: \Lambda \subset \mathbb{Z}\right\}$ by

$$
\Phi_{Y}(\omega)=\left\{\begin{array}{cc}
-h \omega(i) ; & Y=\{i\} \\
-J_{i j} \omega(i) \omega(j) ; & Y=\{i, j\} \\
0 ; & \text { otherwise }
\end{array}\right.
$$

and the Hamiltonian by

$$
\mathfrak{H}_{\Lambda}=\sum_{X \subset \Lambda} \Phi_{X}(\omega)
$$

Here, $\omega(i) \in X$, and $h \in \mathbb{R}$ represents an external magnetic field. In the following, we index all quantities by $N$ instead of $\Lambda$.

Let

$$
J_{i j}=\frac{1}{N} Q\left(\xi_{i} ; \xi_{j}\right)
$$

where $\xi_{i}=\left(\xi_{i 1}, \xi_{2}, \ldots, \xi_{i d}\right)$ are i.i.d. random variables each with a common symmetric distribution denoted by $\mathbb{P}_{\xi}$. Denote by $\underline{\xi}$, the sequence $\left(\xi_{i}\right)_{i=1}^{N}$. We assume that $\mathbb{E}\left(\left|\xi_{i \alpha}\right|\right)<\infty ; 1 \leq \alpha \leq d$ and that $\xi_{i \alpha}$ are i.i.d. random variables. $Q$ is a symmetric function of the $\xi_{i}$ 's which means

$$
{ }^{{ }^{6}}{ }_{N}=\frac{1}{N} \sum_{(i, j)} Q\left(\xi_{i} ; \xi_{j}\right) \omega(i) \omega(j)-h \sum_{i} \omega(i)
$$

where $(i, j)$ represents an index pair.

The partition function is defined by

$$
\begin{aligned}
\mathscr{Z}_{N}(\beta, h)=\sum_{\omega} e^{-\beta^{\mathfrak{J} \mathfrak{G}_{N}}(\omega)} \\
=2^{N} \int_{\Omega_{N}} \exp \left[-\beta \mathfrak{H}_{N}\right] \mathbb{P}_{\mu}(d \omega),
\end{aligned}
$$

where $\beta$ is the inverse temperature.

The range of the interactions tends to $\infty$ as $N \rightarrow \infty$. The specific free energy is defined as

$$
f(\beta, h)=-\frac{1}{\beta} \lim _{N \rightarrow \infty} \frac{1}{N} \ln \mathscr{Z}_{N}(\beta, h) .
$$

We shall prove that the above limit exists $\mathbb{P}_{\xi^{-}}$a.s. in the following case. 


\section{Separable Interactions}

Let $h=0, d=2$, and consider a separable quadratic interaction of the form

$$
Q\left(\xi_{i} ; \xi_{j}\right)=J\left[\xi_{i 1} \xi_{j 2}+\xi_{j 1} \xi_{i 2}\right]
$$

Define the observables

$$
q_{1 N}=\frac{1}{N} \sum_{i=1}^{N} \xi_{i 1} \omega(i), \quad q_{2 N}=\frac{1}{N} \sum_{i=1}^{N} \xi_{i 2} \omega(i),
$$

and define their distribution by the image measure $\mathbb{F}_{N}[2,3]$ where

$$
\mathbb{F}_{N}(A)=\mathbb{P}_{\mu}\left(q_{N}^{-1}(A)\right)
$$

for all $A \in \mathfrak{B}\left(\mathbb{R}^{2}\right)$, under the measurable map $q_{N}=\left(q_{1 N}, q_{2 N}\right): \Omega_{N} \rightarrow \mathbb{R}^{2}$. Notice that $\mathbb{F}_{N}$ depends on the random variables $\xi_{i}$. The partition function $(2.6)$ becomes

$$
\mathscr{Z}_{N}(\beta, 0)=2^{N} \int e^{N K q_{1} q_{2}} \mathbb{F}_{N}(d q)
$$

where $K=\beta J$ and the specific free energy can be written as

$$
f(\beta)-\frac{1}{\beta} \lim _{N \rightarrow \infty} \frac{1}{N} \ln 2^{N} \int_{\mathbb{R}^{2}} e^{n K q_{1} q_{2}} \mathbb{F}_{N}(d q) .
$$

Let $\left\{y_{n} \in \mathbb{R}^{d} ; n=1,2, \ldots\right\}$, where $d \in \mathbb{Z}^{+}$, be a sequence of random variables defined on some probability space $(\Omega, \mathscr{F}, \mathbb{P})$. We define the cumulant generating function by

$$
C(t)=\lim _{n \rightarrow \infty} \frac{1}{n} \ln \mathbb{E}\left[e^{\left\langle t, y_{n}\right\rangle}\right] \quad t \in \mathbb{R}^{d}
$$

where $\mathbb{E}$ denotes the expectation with respect to $\mathbb{P}$, and $\langle-,-\rangle$ denotes the Euclidean inner produce in $\mathbb{R}^{d}$. Our aim is to show the existence of $C(t) \underline{\xi}$-a.e., $\forall t$ and then to use Cramér's Theorem $[14,4]$ to find a candidate for the rate function $I\left(q_{1}, q_{2}\right)$ which would be the Legendre-Fenchel transform [4] of $C(t)$. Then we discuss the $\mathbb{P}_{\xi^{\text {-a.s. }}}$. convergence of (3.6) considering the bounded and unbounded distributions for $\xi$, and also show that the null set, where this convergence is not valid, is independent of $t$. We then apply Varadhan's Theorem [14] to evaluate the limit (3.5).

\subsection{Evaluation of the Free Energy}

Lemma 3.1: Assume $\mathbb{E}\left(e^{s\|\xi\|}\right)<\infty$ for all $s>0$ and $M>\mathbb{E}[\|\xi\|]$. Define closed sets

Then

$$
A_{n}=\left\{\underline{\xi}: X_{n}=\frac{1}{n} \sum_{i=1}^{n}\left\|\xi_{i}\right\| \geq M\right\} .
$$

$$
\mathbb{P}\left(\limsup _{n \rightarrow \infty} A_{n}\right)=0
$$




\section{$\left(\|-\|\right.$ denotes the Euclidean norm in $\mathbb{R}^{d}$.)}

Proof: $\left\|\xi_{i}\right\|$ are independent random variables with the same distribution. By Cramér's Theorem, we have a large deviation property for $X_{n}$ with rate function

$$
I(x)=\sup _{s>0}\{s x-C(s)\}
$$

where

$$
C(s)=\ln \mathbb{E}\left(e^{s\left\|\xi_{i}\right\|}\right)
$$

$C(s)<\infty$ by the assumption. For $A_{n}$ closed, it follows by the large deviation upper bound that

$$
\limsup _{n \rightarrow \infty} \frac{1}{n} \ln \mathbb{P}\left(A_{n}\right) \leq-\inf _{x \geq M} I(x)=:-I_{M}
$$

This implies that $\forall \epsilon>0, \exists n_{0} \in \mathbb{N}$ such that

$$
\begin{gathered}
n \geq n_{0} \Rightarrow \frac{1}{n} \ln \mathbb{P}\left(A_{n}\right) \leq-I_{M}+\epsilon \\
\Rightarrow \mathbb{P}\left(A_{n}\right) \leq e^{-n\left(I_{M}-\epsilon\right)} .
\end{gathered}
$$

Since $A_{n}$ is closed an $\mathbb{E}[\|\xi\|] \notin A_{n}$, we have $I_{M}>I(\mathbb{E}[\|\xi\|])=0$. Choose $\epsilon<I_{M}$ and we get

$$
\sum_{n \geq n_{0}} \mathbb{P}\left(A_{n}\right) \leq \sum_{n \geq n_{0}} e^{-n\left(I_{M}-\epsilon\right)}<\infty .
$$

Now it follows from Borel-Cantelli's Lemma [6] that

$$
\mathbb{P}\left(\limsup _{n \rightarrow \infty} A_{n}\right)=0 .
$$

Theorem 3.1: Let $d=2, \xi_{i}$ be i.i.d. random variables with $\mathbb{E}\left(e^{t\left\|\xi_{i}\right\|}\right)<\infty$ for all $t>0$. Then

(a) for every $t=\left(t_{1}, t_{2}\right) \in \mathbb{R}^{2}$ and $\underline{\xi}$-a.e. the function

$$
C(t)=\lim _{N \rightarrow \infty} \frac{1}{N} \ln \int_{\Omega_{N}} e^{N\left[\left\langle t, q_{N}(\xi)\right\rangle\right]} \mathbb{P}_{\mu}(d \omega)
$$

exists and is independent of $\underline{\xi}$.

(b) For $\underline{\xi}$-a.e., the distributions $\mathbb{F}_{N}$ satisfy the Large Deviation Principle $(L D \bar{P})$ with rate function given by

$$
I(q)=\sup _{t \in \mathbb{R}^{2}}\{\langle t, q\rangle-C(t)\}
$$

(c) The specific free energy of the spin glass model defined by the Hamiltonian (2.5) with the separable interaction (3.1) exists $\underline{\xi}-a . e$. and is given by

$$
f(\beta)=-\frac{1}{\beta}\left(\sup _{q_{1}, q_{2}}\left\{K q_{1} q_{2}-I\left(q_{1}, q_{2}\right)\right\}+\ln 2\right) .
$$


Proof: $(a)$

Write

$$
\begin{gathered}
C(t)=\lim _{N \rightarrow \infty} \frac{1}{N} \ln \int_{\Omega_{N}} \exp \left\{\sum_{i=1}^{N} \omega(i)\left\langle t, \xi_{i}\right\rangle\right\} \mathbb{P}_{\mu}(d \omega) \\
=\lim _{N \rightarrow \infty} \frac{1}{N} \sum_{i=1}^{N} \ln \cosh \left(\left\langle t, \xi_{i}\right\rangle\right) .
\end{gathered}
$$

$$
g_{N}(t, \underline{\xi})=\frac{1}{N} \sum_{i=1}^{N} \ln \cosh \left(\left\langle t, \xi_{i}\right\rangle\right)
$$

and

$$
g(t)=\mathbb{E}_{\xi}\left[\ln \cosh \left(\left\langle t, \xi_{i}\right\rangle\right)\right]
$$

so that $g(t)$ is independent of $i$. We show that there exists a null set $\mathcal{N}$ such that $\forall t \in \mathbb{R}^{2}$ and $\forall \underline{\xi} \notin \mathcal{N}, g_{N}(t, \underline{\xi}) \rightarrow g(t)$, i.e.,

$$
C(t)=\mathbb{E}_{\xi}\left[\ln \cosh \left(\left\langle t, \xi_{i}\right\rangle\right)\right] \quad \underline{\xi} \text { a.e. and } \forall t .
$$

Notice that at a fixed $t, X_{i}=\ln \cosh \left(\left\langle t, \xi_{i}\right\rangle\right)$ are independent random variables with identical distributions and hence it follows from the strong law of large numbers that $g_{N}(t, \underline{\xi}) \rightarrow g(t)$ a.s. This means that for each $t \in D$, there is a null set $\mathcal{N}_{t}$ such that, if $\underline{\xi} \notin \mathcal{N}_{t}$, then

$$
g_{N}(t, \underline{\xi}) \rightarrow g(t)
$$

Let $D \subset \mathbb{R}^{2}$ be a dense countable set and put

$$
\mathcal{N}=\bigcup_{t \in D} \mathcal{N}_{t}
$$

Then $\mathcal{N}$ is also a $\mathbb{P}_{\xi}$ null set since it is a countable union of null sets. If $\underline{\xi} \notin \mathcal{N}$, then we have

$$
g_{N}(t, \underline{\xi}) \rightarrow g(t) \quad \forall t \in D
$$

Let $\epsilon>0$ be given and set $\left\|t-t^{\prime}\right\|<\epsilon / 3 M$ for some $M>\mathbb{E}[\|\xi\|]$. Then

$$
\begin{aligned}
\left|g_{N}(t, \underline{\xi})-g_{N}\left(t^{\prime}, \underline{\xi}\right)\right| & =\frac{1}{N}\left|\sum_{i=1}^{N}\left[\ln \cosh \left(\left\langle t, \xi_{i}\right\rangle\right)-\ln \cosh \left(\left\langle t^{\prime}, \xi_{i}\right\rangle\right)\right]\right| \\
\leq & \left\|t-t^{\prime}\right\| \frac{1}{N} \sum_{i=1}^{N}\left\|\xi_{i}\right\| .
\end{aligned}
$$

Now suppose that $\underline{\xi}$ has bounded distributions. Then, we can find an $M$ such that $\left\|\xi_{i \alpha}\right\| \leq M$ with probability 1 (for example, $\xi_{i \alpha}= \pm 1$ with probability $1 / 2$ ). For unbounded distributions we shall assume $\mathbb{E}\left(e^{t\|\xi\|}\right)<\infty$ (notice that this condition is 
satisfied for Gaussian distributions). By Lemma 3.1, we have

$$
\mathbb{P}\left(\limsup _{n \rightarrow \infty} A_{n}\right)=0 \text {. }
$$

This implies that if $\underline{\xi} \notin \mathcal{A}:=\limsup _{n \rightarrow \infty} A_{n}$, then eventually

$$
\frac{1}{n} \sum_{i=1}^{n}\left\|\xi_{i}\right\|<M \text { a.s. }
$$

So, take $\underline{\xi} \notin \mathcal{N} \cup \mathcal{A}$ when $\underline{\xi}$ has an unbounded distribution and get, for those $\underline{\xi}$,

$$
\left|g_{N}(t, \underline{\xi})-g_{N}\left(t^{\prime}, \underline{\xi}\right)\right| \leq \frac{\epsilon}{3} \text { with probability } 1 \text {. }
$$

Now for an arbitrary $t \in \mathbb{R}^{2}, \underline{\xi} \notin \mathcal{N} \cup \mathcal{A}$ and $t^{\prime} \in D$ with $\left\|t-t^{\prime}\right\|<\epsilon / 3 M$, it follows from (3.25) and the continuity of the function $g$, that,

$$
\begin{gathered}
\left|g_{N}(t, \underline{\xi})-g_{N}(t)\right| \leq\left|g_{N}(t, \underline{\xi})-g_{N}\left(t^{\prime}, \underline{\xi}\right)\right| \\
\quad+\left|g_{N}\left(t^{\prime}, \underline{\xi}\right)-g\left(t^{\prime}\right)\right|+\left|g(t)-g\left(t^{\prime}\right)\right|<\epsilon .
\end{gathered}
$$

We conclude that

$$
g_{N}(t, \underline{\xi}) \rightarrow g(t) \quad \forall t \in \mathbb{R}^{2} \text { and } \mathbb{P}_{\xi^{- \text {a.s. }}}
$$

(b) Since $C(t)$ exists $\underline{\xi}$-a.e. for all $t$ and is a convex function of $t$, we apply Cramér's Theorem $[14,4]$. Since

$$
\left(q_{1 N}, q_{2 N}\right)=\left(\frac{1}{N} \sum_{i=1}^{N} \xi_{i 1} \omega(i), \frac{1}{N} \sum_{i=1}^{N} \xi_{i 2} \omega(i)\right)
$$

is a pair of independent random variables with common distribution function $\mathbb{F}_{N}$, and is in the form of the averages described in Cramér's Theorem, we have

$$
I\left(q_{1}, q_{2}\right)=\sup _{t_{1}, t_{2}}\left\{t_{1} q_{1}+t_{2} q_{2}-C\left(t_{1}, t_{2}\right)\right\} \quad \text { } \xi \text {-a.e. }
$$

(c) Now $\mathbb{F}_{N}$ satisfies the LDP with rate function $I\left(q_{1}, q_{2}\right)$, and $q_{1 N} q_{2 N}$ is a continuous function from $\mathbb{R}^{2} \rightarrow \mathbb{R}$. In order to apply Varadhan's Theorem [14] to evaluate the limit (3.4), we need $K q_{1 N} q_{2 N}$ t be bounded above. This follows from Lemma 3.1:

If $\underline{\xi} \notin \mathcal{A}, \exists N_{0} \in \mathbb{N}$ such that

$$
\frac{1}{N} \sum_{i=1}^{N}\left|\xi_{i}\right|<M \quad N \geq N_{0}
$$

for $N \geq N_{0}$. Hence we get for $N \geq N_{0}$,

$$
q_{1 N} q_{2 N} \leq M^{2} \text { a.s. }
$$

Finally, it follows from Varadhan's Theorem that 


$$
-\beta f(\beta)=\sup _{q_{1} q_{2}}\left\{K q_{1} q_{2}-I\left(q_{1}, q_{2}\right)\right\}+\ln 2 \text { exists } \underline{\xi} \text {-a.e. }
$$

In the following, we determine the maximizers of the free energy functional (3.17), the critical temperature of the spin glass phase transition, and also find expressions for some thermodynamic quantities.

Definition 3.1: Define the specific entropy as a function of the specific internal energy $u$ by

$$
s(u)=\inf _{\beta \geq 0} \beta\{u-f(\beta)\} .
$$

Proposition 3.1: Let $\left(\widetilde{q}_{1}, \widetilde{q}_{2}\right)$ be a maximizer of the free energy functional (3.17) with $\xi=\left(\xi_{1}, \xi_{2}\right)$ and $\eta=\xi_{1}+\xi_{2}$. Also take $J=1$ so that $K=\beta$. Then

(i) $\quad \widetilde{q}_{1}=\widetilde{q}_{2}=\widetilde{q}$ and $\widetilde{q}$ satisfies $2 \widetilde{q}=\mathbb{E}_{\eta}[\eta \tanh \beta \widetilde{q} \eta]$. Furthermore, $\widetilde{q}$ has nonzero solutions if $\beta>\beta_{C}=2 / \mathbb{E}\left[\eta^{2}\right]$ and $\tilde{q}$ for $\beta \leq \beta_{C}$.

(ii) $\quad I(\widetilde{q})=2 \beta \widetilde{q}^{2}-\mathbb{E}_{\eta}[\ln \cosh \beta \widetilde{q} \eta]$.

(iii) $\quad-\beta f(\beta)=\mathbb{E}_{\eta}[\ln \cosh \beta \tilde{q} \eta]-\beta \widetilde{q}^{2}+\ln 2$.

(iv) The specific internal energy and the specific entropy are given by

and

$$
u(\beta)=-\widetilde{q}^{2}
$$

$$
\frac{s(\beta)}{k_{B}}=\mathbb{E}_{\eta}[\ln \cosh \beta \widetilde{q} \eta]-2 \beta \widetilde{q}^{2}+\ln 2
$$

respectively. ( $k_{B}$ is the Boltzmann constant.)

Proof: Since $C(t)$ is differentiable for all $t$, the maximization conditions of (3.16) gives

$$
q_{1}=\frac{\partial C\left(t_{1}, t_{2}\right)}{\partial t_{1}}, q_{2}=\frac{\partial C\left(t_{1}, t_{2}\right)}{\partial t_{2}} \forall q_{1}, q_{2} .
$$

If $\left(\widetilde{q}_{1}, \widetilde{q}_{2}\right)$ is an interior point of the essential domain of $I$, which we shall denote by essdomI, then $I$ is differentiable [4]. For $\left(\widetilde{q}_{1}, \widetilde{q}_{2}\right) \in \operatorname{int}($ ess dom $I)$ we have by (3.29),

$$
\beta \widetilde{q}_{2}=\frac{\partial I\left(q_{1}, q_{2}\right)}{\partial \widetilde{q}_{1}}, \beta \widetilde{q}_{1}=\frac{\partial I\left(q_{1}, q_{2}\right)}{\partial \widetilde{q}_{2}} .
$$

Differentiating (3.16),

$$
\frac{\partial I}{\partial q_{1}}=t_{1}\left(\tilde{q}_{1}, \tilde{q}_{2}\right), \frac{\partial I}{\partial q_{2}}=t_{2}\left(\widetilde{q}_{1}, \tilde{q}_{2}\right) .
$$

hence we get $t_{1}\left(\widetilde{q}_{2}\right)=\beta \widetilde{q}_{2}, t_{2}\left(\widetilde{q}_{1}\right)=\beta \widetilde{q}_{1}$. Differentiating (3.20) with respect to $t_{1}$ and $t_{2}$, we have

$$
\begin{aligned}
& \tilde{q}_{1}=\mathbb{E}_{\xi}\left[\xi_{1} \tanh \beta\left(\xi_{1} \tilde{q}_{2}+\xi_{2} \widetilde{q}_{1}\right)\right] \\
& \tilde{q}_{2}=\mathbb{E}_{\xi}\left[\xi_{2} \tanh \beta\left(\xi_{1} \widetilde{q}_{2}+\xi_{2} \widetilde{q}_{1}\right)\right] .
\end{aligned}
$$

We now prove $(i)$.

Write

$$
t_{1} \xi_{1}+t_{2} \xi_{2}=\frac{1}{2}\left(t_{1}+t_{2}\right)\left(\xi_{1}+\xi_{2}\right)+\frac{1}{2}\left(t_{1}-t_{2}\right)\left(\xi_{1}-\xi_{2}\right)
$$


and

$$
q_{1} q_{2}=\frac{1}{4}\left(p_{1}^{2}-p_{2}^{2}\right)
$$

where $p_{1}=q_{1}+q_{2}$ and $p_{2}=q_{1}-q_{2}$. Then $I$ can be written as

$$
I\left(p_{1}, p_{2}\right)=\sup _{s_{1}, s_{2}}\left\{\frac{1}{2} s_{1} p_{1}+\frac{1}{2} s_{2} p_{2}-C\left(\frac{s_{1}+s_{2}}{2}, \frac{s_{1}-s_{2}}{2}\right)\right\}
$$

where $s_{1}=t_{1}+t_{2}$ and $s_{2}=t_{1}-t_{2}$. Then (3.29) becomes

$$
\begin{gathered}
-\beta f(\beta)-\ln 2=\sup _{q_{1}, q_{2}}\left\{\beta q_{2} q_{2}-I\left(q_{1}, q_{2}\right)\right\} \\
=\sup _{p_{1}, p_{2}}\left\{\frac{1}{4} \beta p_{1}^{2}-\frac{1}{4} \beta p_{2}^{2}-I\left(p_{1}, p_{2}\right)\right\} \\
=\sup _{p_{1}}\left\{\frac{1}{4} \beta p_{1}^{2}-\inf _{p_{2}}\left\{\frac{1}{4} \beta p_{2}^{2}+I\left(p_{1}, p_{2}\right)\right\}\right\} .
\end{gathered}
$$

Since $I\left(p_{1}, p_{2}\right)=I\left(p_{1},-p_{2}\right)$, the above infimum must be attained when $p_{2}=q_{1}-$ $q_{2}=0$. Substituting this in (3.34) and adding we find

$$
2 \widetilde{q}=\mathbb{E}_{\eta}(\eta \tanh (\beta \widetilde{q} \eta))
$$

Differentiating the right-hand side of (3.38) gives

and we have

$$
\frac{d}{d q} \mathbb{E}[\eta \tanh (\beta q \eta)]=\beta \mathbb{E}\left[\frac{\eta^{2}}{\cosh ^{2}(\beta q \eta)}\right],
$$

$$
0<\beta \mathbb{E}\left(\frac{\eta^{2}}{\cosh ^{2}(\beta q \eta)}\right) \leq \beta \mathbb{E}\left(\eta^{2}\right)
$$

Hence, $\mathbb{E}(\eta \tanh \beta \widetilde{q} \eta)$ is increasing and its derivative is bounded above by $\beta \mathbb{E}\left(\eta^{2}\right)$. It follows that if $\beta \mathbb{E}\left(\eta^{2}\right)>2$, then (3.38) has three solutions, two of which are non-zero. It is easy to check that the supremum in (3.37) is then attained for $p_{1}=2 \widetilde{q}$, where $\tilde{q}$ is either non-zero solution. If $\beta \mathbb{E}\left(\eta^{2}\right) \leq 2$, then $\tilde{q}=0$ is the only solution. We conclude that the critical inverse temperature is

$$
\beta_{C}=\frac{2}{\mathbb{E}\left(\eta^{2}\right)}
$$

(ii) Take $\tilde{q}_{1}=\tilde{q}_{2}=\tilde{q}$. We can write the rate function (3.16), using (3.32) and $(i)$, as

$$
I(\widetilde{q}, \widetilde{q})=2 \beta \widetilde{q}^{2}-\mathbb{E}_{\eta}[\ln \cosh \beta \widetilde{q} \eta] .
$$

(iii) Substituting (ii) in (3.17), we get 


$$
-\beta f(\beta)=\mathbb{E}_{\eta}[\ln \cosh \beta \tilde{q} \eta]-\beta \widetilde{q}^{2}+\ln 2 .
$$

(iv) The specific internal energy is determined by minimizing (3.30) as

$$
u(\beta)=\frac{d}{d \beta}(\beta f(\beta))=-q^{2}(\beta)
$$

and the specific entropy by

$$
\frac{s(\beta)}{k_{B}}=\beta(u(\beta)-f(\beta))
$$

which gives

$$
\frac{s(\beta)}{k_{B}}=\mathbb{E}_{\eta}[\ln \cosh \beta \widetilde{q} \eta]-2 \beta \widetilde{q}^{2}+\ln 2
$$

Remark 3.1: 1 . Notice the low temperature limit of $(3.38)$ is

$$
\tilde{q}= \pm \frac{1}{2} \mathbb{E}_{\eta}[|\eta|]
$$

since $\tanh [\beta q \eta] \rightarrow \operatorname{sign}(q \eta)$ as $\beta \rightarrow \infty$.

2. For $\beta \leq \beta_{C}, s=k_{B} \ln 2$.

3. The solutions of $\widetilde{q}$ for $\beta>\beta_{C}$ have to be obtained by numerically solving the implicit equation (3.38). In the following, we consider the solutions with specific distributions in more detail.

Proposition 3.2: (a) Let $\xi_{1}, \xi_{2}$ each have a general symmetric discrete distribution and let $\eta=\xi_{1}+\xi_{2}$. Then, as $\beta \rightarrow \infty$,

$$
s \rightarrow k_{B} \mathbb{P}(\eta=0) \ln 2
$$

(b) Let $\xi_{1}, \xi_{2}$ have a standard normal distribution. Then $\tilde{q} \rightarrow 1 / \sqrt{\pi}$ and $s \rightarrow 0$ as $\beta \rightarrow \infty$.

Proof: $(a)$ By $(3.45)$ with $k_{B}=1$ we have $s(\beta)=\mathbb{E}_{\eta}[\ln \cosh \beta \tilde{q} \eta]-2 \beta \widetilde{q}^{2}+\ln 2$. As $\beta \rightarrow \infty, \ln \cosh (\beta \tilde{q} \eta) \sim \beta|\tilde{q} \eta|-\ln 2$. However, irrespective of $\beta$, when $\eta=0$, $\ln \cosh (\beta \tilde{q} \eta)=0$. To compensate, we add the term $\mathbb{P}(\eta=0) \ln 2$. Hence, as $\beta \rightarrow \infty$,

$$
\mathbb{E}_{\eta}[\ln \cosh (\beta \tilde{q} \eta)] \rightarrow \mathbb{E}_{\eta}[\beta|\tilde{q} \eta|]-\ln 2+\mathbb{P}(\eta=0) \ln 2
$$

Using the above and (3.46) we get

$$
\lim _{\beta \rightarrow \infty} s(\beta)=\mathbb{P}(\eta=0) \ln 2 .
$$

(b) Denote $\left.q\right|_{T=0}$ by $q_{0}$. Then from (3.46)

$$
q_{0}=\frac{1}{2} \mathbb{E}\left(\left|\xi_{1}+\xi_{2}\right|\right)=\frac{1}{\sqrt{2}} \mathbb{E}_{\eta}(|\eta|)=\frac{1}{\sqrt{2}} \int_{-\infty}^{\infty}|\eta| e^{-\eta^{2} / 2} \frac{d \eta}{\sqrt{2 \pi}}=\frac{1}{\sqrt{\pi}} \text {. }
$$

Next we show that

(i) $\quad \liminf _{\beta \rightarrow \infty} s(\beta) \geq 0$ :

$$
\liminf _{\beta \rightarrow \infty} s(\beta)=\liminf _{\beta \rightarrow \infty}\left\{\mathbb{E}_{\eta}[\ln \cosh (\beta \widetilde{q} \eta)]-2 \beta \widetilde{q}^{2}+\ln 2\right\}
$$




$$
\begin{gathered}
\geq \liminf _{\beta \rightarrow \infty}\left\{\mathbb{E}_{\eta}\left[\beta q_{0}|\eta|-\ln 2\right]-2 \beta q_{0}^{2}+\ln 2\right\} \\
=\liminf _{\beta \rightarrow \infty}\left(\beta q_{0} \mathbb{E}_{\eta}[|\eta|]-2 \beta q_{0}^{2}\right)=0
\end{gathered}
$$

and

(ii) given $\delta>0,(\delta<\ln 2)$, limsup $\sup _{\beta \rightarrow \infty} s(\beta) \leq \delta$ :

Denote $f(\eta, \beta)=\ln \cosh \left(\beta q_{0} \eta\right)$ and $g(\eta, \bar{\beta})=\left|\beta q_{0} \eta\right|-\ln 2$.

Choose $x(\delta)$ such that $f(\eta, \beta)-g(\eta, \beta) \leq \delta$ for all $\eta \geq x(\delta) / \beta$. This implies that

$$
x(\delta)=\frac{1}{2 q_{0}} \ln \frac{1}{e^{\delta}-1} .
$$

We divide the range $\mathbb{R}$ of $\eta$ into the two intervals $I_{x, \beta}=[-x / \beta, x / \beta]$ and $I_{x, \beta}^{C}$. Approximating $f(\eta, \beta)$ by $g(\eta, \beta)$ on $I_{x \beta}^{C}$, then

$$
\begin{gathered}
\limsup _{\beta \rightarrow \infty}(\beta)=\limsup _{\beta \rightarrow \infty}\left\{\mathbb{E}_{\eta}\left[\ln \cosh \left(\sqrt{2} \beta q_{0} \eta\right)\right]-2 q_{0}^{2} \beta+\ln 2\right\} \\
\leq \limsup _{\beta \rightarrow \infty}\left\{\mathbb{E}_{\eta}\left[\left|\beta q_{0} \eta \sqrt{2}\right|-\ln 2+\delta\right]-2 q_{0}^{2} \beta+\ln 2\right\}
\end{gathered}
$$

$$
\leq \delta
$$

On $I_{x, \beta}$, we have by $(3.45)$

$$
\begin{gathered}
\limsup _{\beta \rightarrow \infty}(\beta) \leq \limsup _{\beta \rightarrow \infty}\left\{\mathbb{E}_{\eta}\left[-\ln 2+\left|\sqrt{2} \beta q_{0} \eta\right|+\ln \left(1+e^{-2 \sqrt{2} \beta\left|q_{0} \eta\right|}\right)\right]\right. \\
\left.-2 \beta q_{0}^{2}+\ln 2\right\}
\end{gathered}
$$

where $\eta=\left(\xi_{1}+\xi_{2}\right) / \sqrt{2}$ which also has a standard normal distribution. We evaluate the right-hand side of the above inequality taking $\mu$ as the Gaussian measure:

$$
\begin{gathered}
\limsup _{\beta \rightarrow \infty}(\beta) \leq \limsup _{\beta \rightarrow \infty} \int_{I_{x, \beta}} \ln \left(1+e^{-2 \sqrt{2} \beta q_{0} \eta}\right)_{\mu}(d \eta) \\
\leq \limsup _{\beta \rightarrow \infty} \ln \left(1+e^{2 \sqrt{2} q_{0} x}\right) \frac{2 x}{\beta} \\
=0 .
\end{gathered}
$$

Thus $\lim \sup _{\beta \rightarrow \infty} s(\beta) \leq \delta$ for any $\delta>0$ for all $\eta \in \mathbb{R}$.

From $(i)$ and $(i i)$ it follows that $\lim _{\beta \rightarrow \infty} s(\beta)=0$.

Example 1: Let $\xi_{1}, \xi_{2}$ have the same symmetric discrete distribution given by $\xi_{1}, \xi_{2}=i ; i \in\{-L,-L+1, \ldots, L\}$ with probability $p_{L}$, where

$$
p_{L}=\frac{1}{2 L+1} \text {. }
$$

Then we have 


$$
\mathbb{P}\left(\xi_{1}+\xi_{2}=\eta\right)=\frac{1+2 L-|\eta|}{(1+2 L)^{2}}=: p_{\eta}
$$

and by Proposition 3.2, we find $\left.s\right|_{T=0}=\ln 2 /(1+2 L)$.

The entropy, computed using (3.45) at several values of $L$ for the above discrete distribution as well as the case where $\xi$ 's have a Gaussian distribution, is plotted as a function of the temperature $T$ in Figure 3.1. In the next section, we compare this entropy with a simulation of this model using the method of coincidence counting [7].

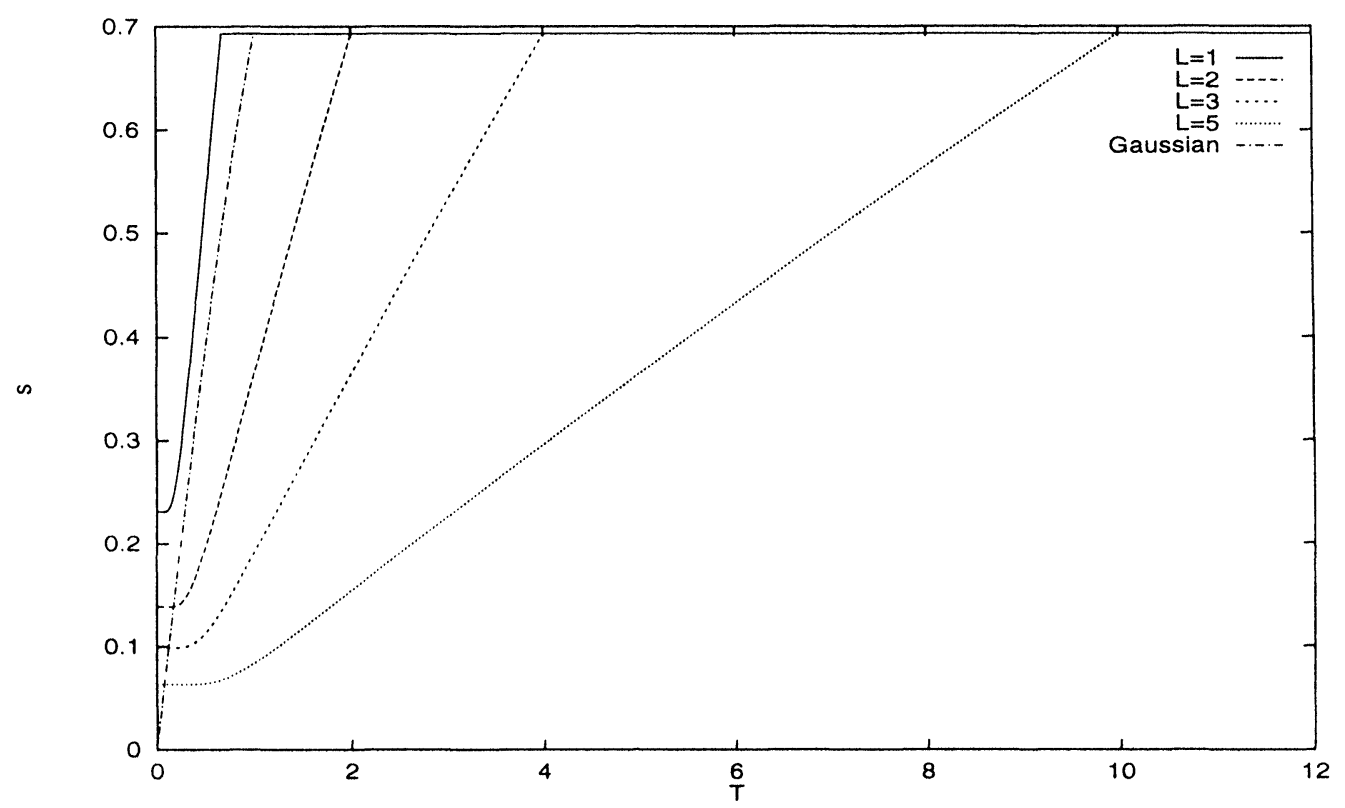

Figure 3.1: Entropy computed via (3.45) for a standard normal distribution and for discrete distributions (3.53)

\section{Entropy of the Model for Discrete Distributions}

We consider the symmetric discrete distribution which was introduced in Example 1 for $L=3$. The method of coincidence counting is used for computing the entropy in a Monte-Carlo simulation [1] introduced by S.K. Ma [7]. The method involves counting the number of states coinciding with a given state where a state is a configuration of the Ising spins. Two states are "coincident" if they do not differ by more than $m$ spins $(m=0,1, \ldots)$, (when $m=0$ the states are identical), and their energies are approximately the same. A detailed discussion about the algorithm can be found in $[7]$.

We compute the analytical result for 12 Ising spins for this distribution by generating all possible $2^{12}$ configurations, and compare this with our simulation (see Figure 4.1). We also plot the entropy in the thermodynamic limit as was done in Figure 3.1 It becomes clear that 12 spins are still far removed from th thermodynamic limit. Indeed, 12 Ising spins are not sufficient to obtain a non-random limit for the entropy. 
With $L=3$, we have 7 possible random values for $\xi$ to appear in a sequence of 22 elements (each spin is connected to 11 others, and each bond has two $\xi$-values). In fact, there are fluctuations in the simulation as well as the analytical result (for 12 spins) between different runs of the program. These random fluctuations cause the slight discrepancy between the simulated and the analytical graphs.

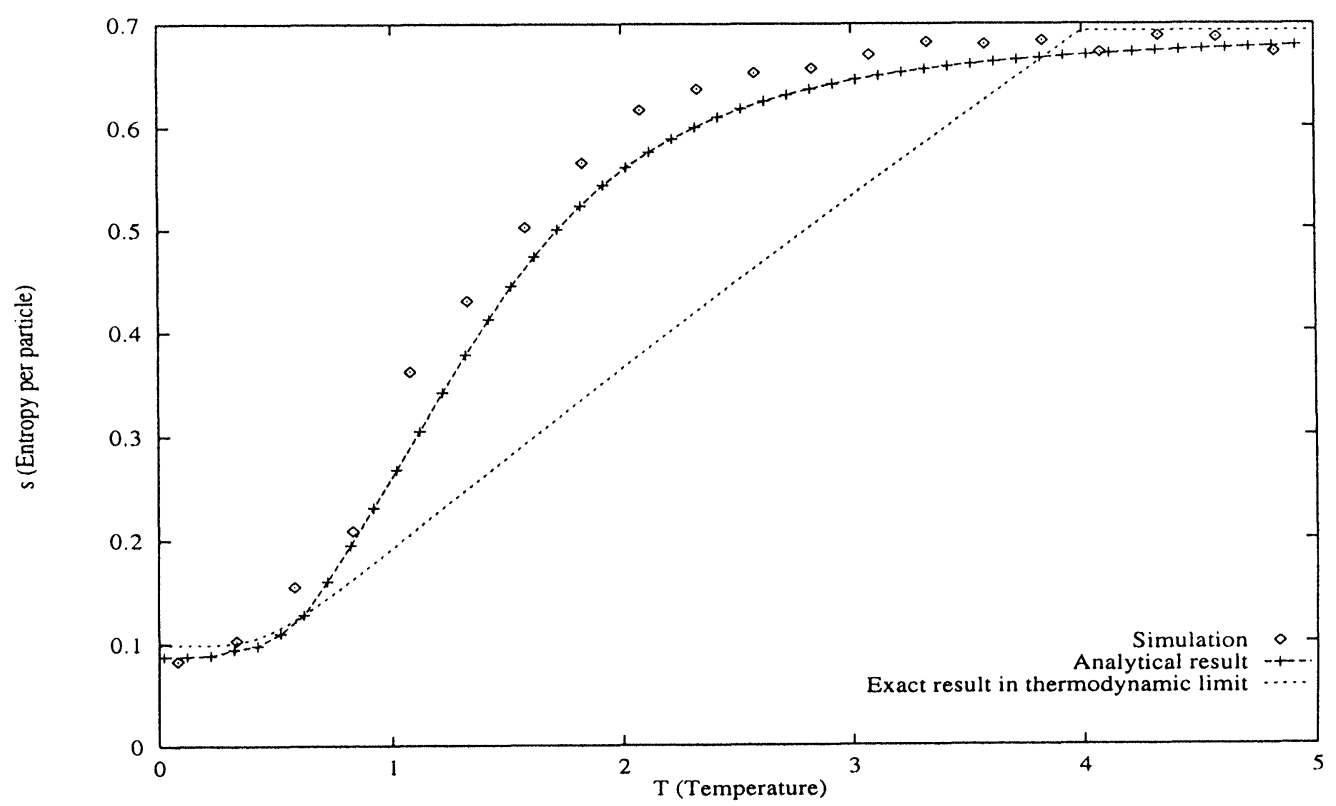

Figure 4.1: Comparison of entropy simulated using the method of coincidence counting for 12 Ising spins with the analytical results for $N=12$ and in the limit $N \rightarrow \infty$.

\section{References}

[1] Binder, K., Monte Carlo Methods in Statistical Physics, Springer-Verlag, Berlin 1979.

[2] Dorlas, T.C., Lecture Notes on Measures on Topological Spaces 1997 (unpublished).

[3] Dorlas, T.C., Lewis, J.T. and Pulé, J.V., Helvetica Physica Acta 64 (1991), 1200.

[4] Ellis, R.S., Entropy, Large Deviations and Statistical Mechanics, SpringerVerlag, Berlin 1985.

[5] Hopfield, J.J., Proc. Natl. Acad. Sci. USA 79 (1982), 2554.

[6] Lamperti, J., Probability, W.A. Benjamin 1966.

[7] Ma, S., J. Stat. Phys. 26 (1981), 2.

[8] Pastur, L.A. and Figotin, A.L., Sov. J. Low. Temp. Phys. 3:6 (1977), 378.

[9] Pastur, L.A. and Figotin, A.L., Theor. Math. Phys. 35 (1978), 403. 
[10] Pastur, L.A. and Figotin, A.L., Theor. Math. Phys. 51 (1982), 564.

[11] van Hemmen, J.L., Phys. Rev. A 34 (1986), 3435.

[12] van Hemmen, J.L., Grensing, D., Huber, A., and Kuhn, R., Z. Phys. B 65 (1986), 53.

[13] van Hemmen, J.L., van Enter, A.C.D., and Canisius, J., Z. Phys. B 50 (1983), 311.

[14] Varadhan, S.R.S., Large Deviations and Applications, SIAM 1984. 


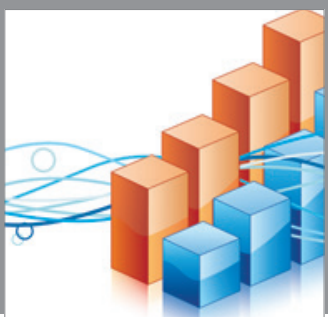

Advances in

Operations Research

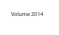

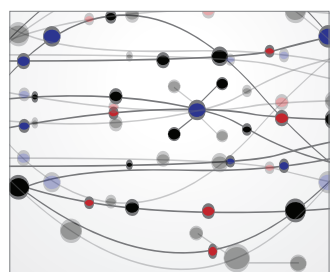

\section{The Scientific} World Journal
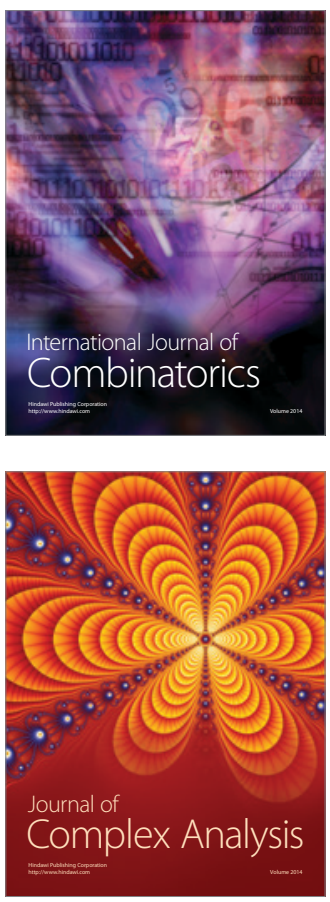

International Journal of

Mathematics and

Mathematical

Sciences
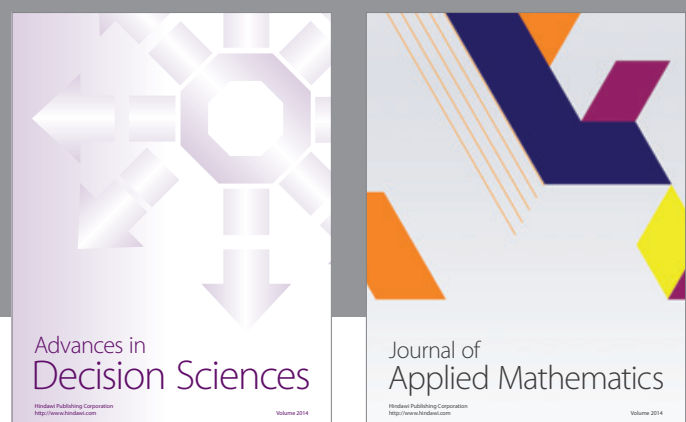

Journal of

Applied Mathematics
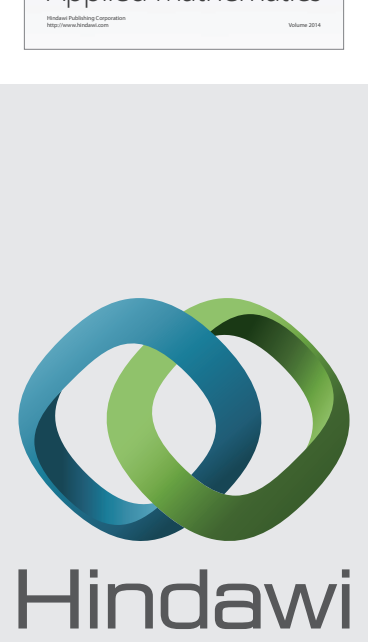

Submit your manuscripts at http://www.hindawi.com
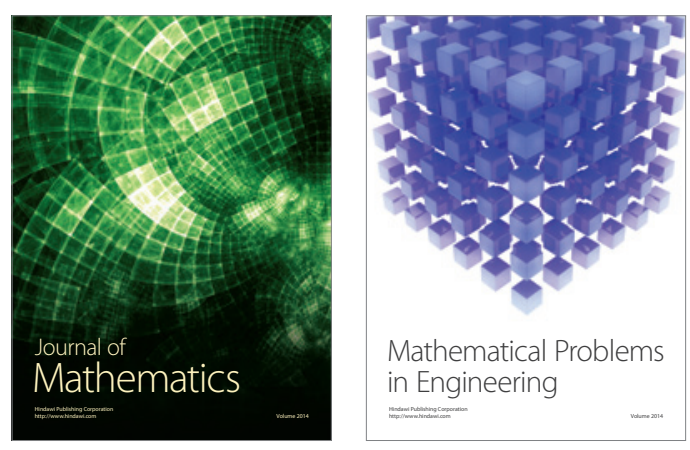

Mathematical Problems in Engineering
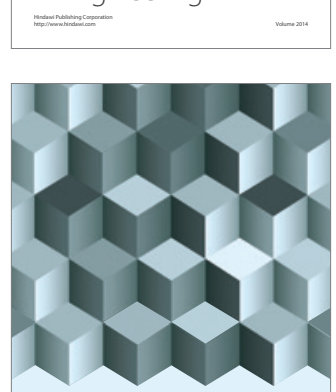

Journal of

Function Spaces
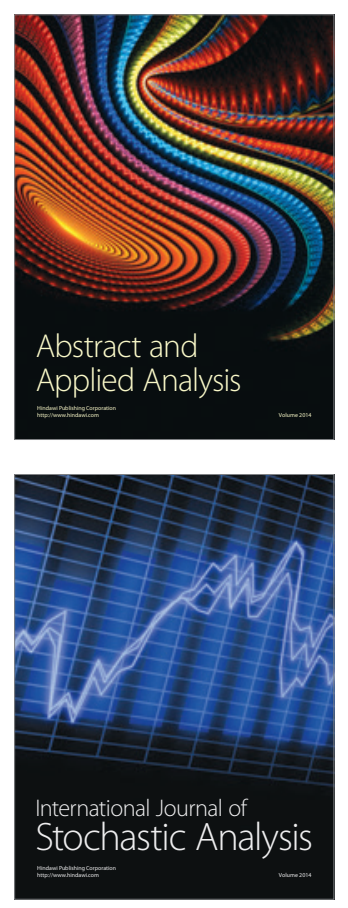

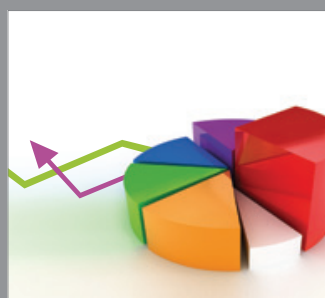

ournal of

Probability and Statistics

Promensencen
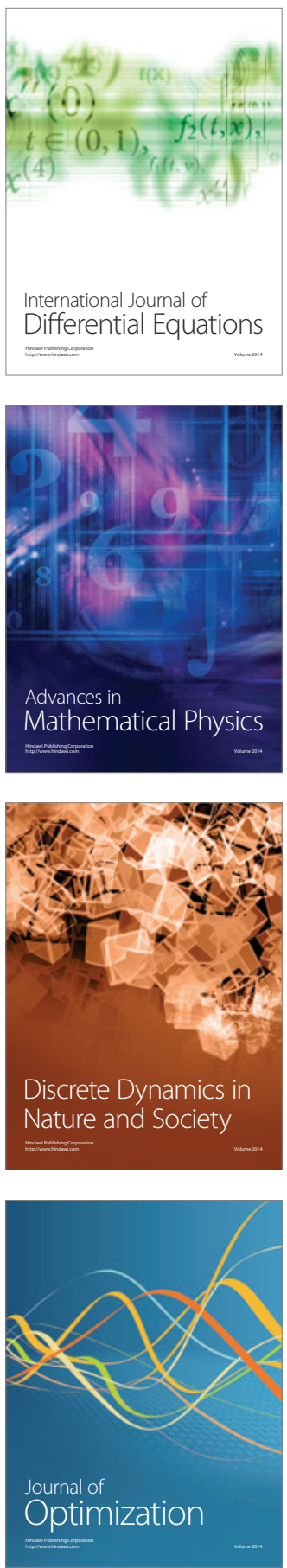